\title{
Assessment of the performance of different element types for guided wave simulations in abaqus
}

Cite as: AIP Conference Proceedings 2309, 020010 (2020); https://doi.org/10.1063/5.0034687

Published Online: 30 November 2020

Ilias N. Giannakeas, Z. Sharif-Khodaei, and M. H. Aliabadi

\section{ARTICLES YOU MAY BE INTERESTED IN}

On the estimation of material properties using guided wave measurements for the calibration of finite element models

AIP Conference Proceedings 2309, 020008 (2020); https://doi.org/10.1063/5.0034686

Synchronized wireless sensors for aircraft structural health monitoring

AIP Conference Proceedings 2309, 020011 (2020); https://doi.org/10.1063/5.0033952

Numerical simulation of guided waves propagation in loaded composite structures AIP Conference Proceedings 2309, 020029 (2020); https://doi.org/10.1063/5.0033968

Challenge us.

What are your needs for periodic signal detection?

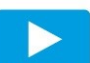

Watch

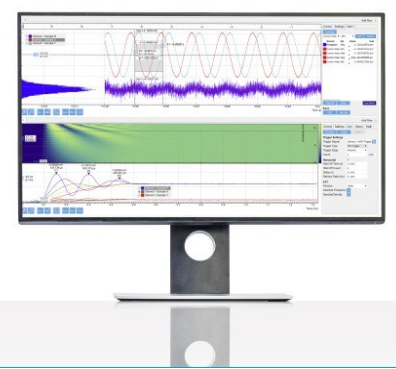

- Zurich Instruments 


\title{
Assessment of the Performance of Different Element Types for Guided Wave Simulations in Abaqus
}

\author{
Ilias N. Giannakeas, ${ }^{1,}$, Z. Sharif-Khodaei ${ }^{1, \text { b) }}$ and M. H. Aliabadi ${ }^{1, c)}$ \\ ${ }^{I}$ Department of Aeronautics, Imperial College London South Kensington Campus, Exhibition Road, SW7 2AZ, \\ London, UK \\ ${ }^{a}$ Corresponding author: i.giannakeas19@imperial.ac.uk, b) z.sharif-khodaei@imperial.ac.uk, \\ c)m.h.aliabadi@imperial.ac.uk
}

\begin{abstract}
Accurate numerical tools for the simulation of wave propagation are essential for the development and optimization of guided wave based Structural Health Monitoring systems. This article aims in delivering a systematic comparison of the Finite Element simulation of Lamb wave propagation using different types of elements. The numerical simulations are all realized within the environment of the commercial software Abaqus. In total three different element types are considered: conventional shell, continuum shell and 3D solid elements. To evaluate the performance of each element type, the numerically simulated signals are compared with experimental measurements from two panels. The first panel is made of Aluminium while the second is a layered composite panel. When continuum or 3D solid elements are used, the numerical predictions are closely correlated to the experimental observations. Accurate predictions were also made using conventional shell elements to model wave propagation in the first panel however, the group velocity for the first symmetric wave mode is over-estimated for the second panel when the excitation frequency is $f_{c}=250 \mathrm{kHz}$.
\end{abstract}

\section{INTRODUTION}

Structural Health Monitoring (SHM) systems are capable of reducing the overall maintenance cost of a structure by regularly performing structural interrogations to identify faults over long distances $(1,2)$. SHM systems allow operators to adopt condition-based maintenance schemes instead of the common schedule-based maintenance, increasing the safety and reliability of the structure while reducing the maintenance costs (3). Guided Wave based SHM systems employ piezoelectric transducers (PZT) for the actuation and sensing of propagating pulses and use signal processing tools to detect changes in the state of the structure $(4,5)$. Such algorithms correlate signals from the pristine state of the structure with the current measurements and use decision-based criteria for fault detection.

Accurate analysis tools for the modeling and simulation of Lamb wave propagation in complex structures is of outmost importance for the design and development of SHM systems. Such tools can be used for the optimization of the sensor placement, evaluation of the systems performance for different types of damage and severity levels and tuning of the excitation signal $(1,2,6)$. Furthermore, they can reduce the requirement of large scale experiments that can be both costly and time consuming (7). Engineers thus require precise information on the characteristics of Lamb wave propagation.

In order to perform Lamb wave analyses, the equation of motion can be solved analytically, semi-analytically or numerically (8). In certain cases, analytical solution of the equation of motion is possible however, when the geometry, boundary conditions or material behaviour are not simple, the problem becomes intractable and closed form solutions are not possible. Numerical techniques can be used in such cases to approximate the solution of the equation of motion. Commonly used numerical techniques include the finite difference method (9), the boundary element method $(10,11)$, the spectral element method $(12,13)$, the finite element method $(2,8,14,15)$, the local interaction simulation approach (LISA) $(16,17)$ and the elastodynamic finite integration technique (EFIT) (18). As noted in (14), the finite element method has been employed by many commercially available software packages and has met wide applicability to real aircraft structures due to its ability to handle complex geometry and composite lay-ups. 
The aim of the present study is to simulate Lamb wave propagation using the commercial finite element package Abaqus. Specifically, the numerical simulation is carried out using central difference integration scheme through the Abaqus/Explicit module. Different element types are available in Abaqus' library that can be used for Lamb wave simulation. Here the conventional shell, continuum shell and 3D solid elements are used for the time domain simulation of wave propagation and the results are compared with experimental measurements taken from an Aluminium and a composite panel.

The present study is structured as follows: Section 2 includes the description of the experimental set-up used for the acquisition of the experimental measurements. The definitions of the numerical models used is presented in Section 3 for all types of elements used. The numerical results and their comparison with the experimental measurements is included in Section 4 along with the discussion of the results. Finally, concluding remarks and recommendations for future work is included in Section 5.

\section{EXPERIMENTAL SET-UP}

In order to validate the performance of the numerical results, experimental measurements are collected from isotropic and anisotropic specimens. The experimentally obtained signals are then compered with the predictions of the numerical simulations.

Circular DuraAct PIC255 piezoelectric transducers are used for both the actuation and the sensing of Lamb waves. The PZTs are surface mounted onto the specimens and bonded through the use of adhesive film. The thickness and the radius of the PZT wafers is $t_{P Z T}=0.5 \mathrm{~mm}$ and $r_{P Z T}=5 \mathrm{~mm}$, respectively. The mechanical properties of the PZTs are Poisson's ratio $v_{P Z T}=0.31$, Young's Modulus $E_{P Z T}=72.5 \mathrm{GPa}$, density $\rho_{P Z T}=7800 \mathrm{~kg} \cdot \mathrm{m}^{-3}$, dielectric constant $K_{3}=1280$ and piezoelectric strain constant $d_{31}=-130 \cdot 10^{-12} \mathrm{mV}^{-1}$.

In total, two panels are used for the acquisition of experimental measurements. The geometry and the location of the PZTs is illustrated schematically in Fig. 1 (A). The first panel (P1), is rectangular with $L=H=287 \mathrm{~mm}$ and it is made out of $\mathrm{Al} \mathrm{6061}$. The sensors are located at a distance $l_{1}=l_{2}=40 \mathrm{~mm}$ from the sides of the panel. The mechanical properties of $\mathrm{Al} 6061$ are listed in Table 1 (19).
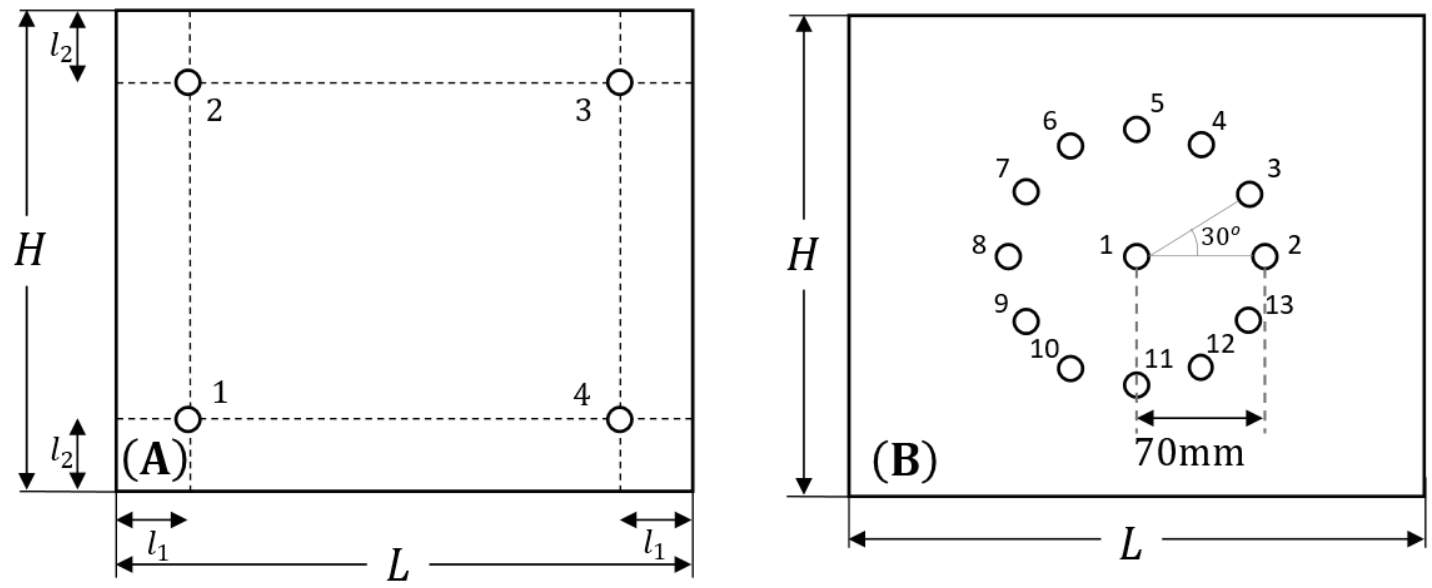

FIGURE 1: Schematic illustration of the geometry and PZT locations for A) Aluminium panel and B) composite panel.

TABLE 1: Mechanical properties of Al 6061

\begin{tabular}{ccc}
\hline$E[\mathbf{G P a}]$ & $\boldsymbol{v}$ & $\boldsymbol{\rho}\left[\frac{\mathbf{k g}}{\mathbf{m}^{\mathbf{3}}}\right]$ \\
\hline 69 & 0.312 & 1600 \\
\hline
\end{tabular}

The second panel (P2), is made using 12 layers of standard thermoset unidirectional prepreg material M21/194/34\%/T800S with stacking sequence $\left[ \pm 45 / 0_{2} / 90 / 0\right]_{S}$. The thickness of each layer is $t_{\text {layer }}=0.184 \mathrm{~mm}$ and the total thickness of the lamina is $t_{l m t}=2.208 \mathrm{~mm}$. The horizontal and vertical dimensions of $\mathrm{P} 2$ are $L=$ $300 \mathrm{~mm}$ and $H=225 \mathrm{~mm}$, respectively, and the $0^{\circ}$ fibre direction is oriented along the horizontal dimension $L$. The 
sensors are placed in a circular pattern near the centre of the panel, as illustrated in Fig. 1 (B). This set-up is chosen to take measurements along different propagation paths and capture the directional dependence of the propagation speed to the propagation direction. This dependence is due to the anisotropic behaviour of the composite panel. Each layer of the prepreg is assumed to behave as an orthotropic material and the mechanical properties used are listed in Table 2 .

TABLE 2: Mechanical properties of the unidirectional M21/194/34\%/T800S prepreg.

\begin{tabular}{cccccccccc}
\hline $\boldsymbol{E}_{\mathbf{1 1}}[\mathrm{GPa}]$ & $\boldsymbol{E}_{\mathbf{2 2}}[\mathrm{GPa}]$ & $\boldsymbol{E}_{\mathbf{3 3}}[\mathrm{GPa}]$ & $\boldsymbol{v}_{\mathbf{1 2}}$ & $\boldsymbol{v}_{\mathbf{1 3}}$ & $\boldsymbol{v}_{\mathbf{2 3}}$ & $\boldsymbol{G}_{\mathbf{1 2}}[\mathrm{GPa}]$ & $\boldsymbol{G}_{\mathbf{1 3}}[\mathrm{GPa}]$ & $\boldsymbol{G}_{\mathbf{2 3}}[\mathrm{GPa}]$ & $\boldsymbol{\rho}\left[\frac{\mathrm{kg}}{\mathbf{m}^{\mathbf{3}}}\right]$ \\
\hline 140.2 & 8.675 & 8.675 & $\begin{array}{c}0.31 \\
2\end{array}$ & $\begin{array}{c}0.31 \\
2\end{array}$ & 0.4 & 4.29 & 4.29 & 3.1 & 1600 \\
\hline
\end{tabular}

In anisotropic material, such as layered composites, Lamb wave propagation is dispersive in nature and the group velocity $c_{g}$ of the wave packet is not the same as the phase velocity $c_{p}$. For this reason, narrowband excitation is in general preferred as it is easier to distinguish the different wave modes while dispersion of the different frequencies is reduced (2). The actuation used is a 5-tone Hanning windowed input pulse given as:

$$
V(t)=A_{0}\left[H(t)-H\left(t-n / f_{c}\right)\right] \sin \left(2 \pi f_{c} t\right)\left(1-\cos \left(2 \pi f_{c} / n\right)\right),
$$

where, $H$ is the Heaviside function, $n$ is the number of cycles in the tone-burst, $f_{c}$ is the central frequency and $A_{0}$ is the amplitude of the input pulse. The amplitude of the actuation is $V_{A m p l}=12 \mathrm{~V}$ measured from peak to peak, applied to the actuator PZT though the use of a National Instrument waveform generator. The response is recorded at the sensor PZT locations using a PXI 5105 Oscilloscope with sampling frequency of 60MHz. The signals are recorded for a total duration of $t_{\text {tot }}=4 \cdot 10^{-4} \mathrm{~s}$. Furthermore, to reduce the measurement noise and improve the signal to noise ratio, each measurement is repeated 30 times and averaged. Each time one PZT acts as the actuator while the rest of the PZTs as sensors.

\section{DEFINITION OF THE NUMERICAL MODELS}

All models considered in this study have been developed within the commercial finite element software Abaqus. Three different elements are considered: i) conventional shell elements, ii) continuum shell elements and iii) solid elements. The aim of the comparison is the investigate the performance of each element type and compare it with experimentally obtained measurements for both the Aluminium (isotropic) and the composite (anisotropic) cases.

Conventional shells use plate theory, which is a simplification of the 3D elasticity, and are suitable for structures with thickness smaller than the other dimensions (20). A reference surface us used to define the geometry of the structure while suitable stress and displacement assumptions are used through the thickness (21). Equivalent stiffness properties are used to describe the behaviour of the material at the reference surface. In composites, the stacking sequence is represented by an equivalent single layer material with a complex constitutive behaviour. Such an example is the Classical Laminated Plate Theory based on Kirchhoff displacements which assumes that the normal remains orthogonal to the middle plane after deformation and neglects shear deformation $(20,22)$. In composite laminates however, neglecting the shear deformation can lead to erroneous results. Shear deformation effects can be included in the formulation using the Reissner-Mindlin plate theory that assumes that the normal to the plate do not remain orthogonal $(20,22)$. However, the transverse shear is assumed constant through the thickness and a Shear Correction Factor (SCF) is required. Finally, higher order theories have been proposed (see e.g. (23)) where higher order expansions of the displacement field are used. Here, the first shear plate theory is adopted and the element S4R from Abaqus' element library is used which is a 4-noded quadrilateral element with 6 degrees of freedom (dofs) per node (three displacement and three rotational).

Contrary to conventional shell elements, continuum shell elements and 3D solid elements discretize the entire geometry of the structure and the thickness of the plate is derived by the elements' nodal positions. Continuum shells implements the kinematic and constitutive behaviour of the conventional shells described above, while solid elements approximate the solution of 3D elasticity $(20,24)$. The elements SC8R and C3D8R from Abaqus' element library are used for the discretization of the continuum shell and solid models, respectively. Both elements are 8-noded quadrilateral elements with 3 displacement dofs per node. However, in order to accurately represent a layered composite material, multiple elements through the thickness might be required. This can lead to a steep increase of the required computational cost to complete the analysis. 
The actuation, propagation and sensing of guided waves is simulated numerically using the three elements described above for the discretization of the panels. In all cases, the PZT wafers are modelled using solid elements (C3D8R). The bonding is assumed perfect and it is enforced by applying kinematic constrains to the bottom surface of the PZT and the top surface of the panel (25). Lamb wave actuation is performed by converting the input voltage from Eq. 1 to an effective displacement that is applied radially at the top circumference of the actuator $(15,26)$. Following (15), the effective displacement is computed as:

$$
d_{r}=Q_{A} V_{\text {Input }},
$$

where, $Q_{A}$ is a conversion constant for the actuation. Similarly, the voltage in the sensor PZTs can be approximated through the strains measurements as:

$$
V_{\text {Output }}=Q_{S} \varepsilon_{c e n}
$$

where, $Q_{S}$ is a conversion constant for the sensing and $\varepsilon_{c e n}$ is the mean strain at the centre of the PZT wafer. The application of the effective radial displacement on the PZT is illustrated schematically in Fig. 2.

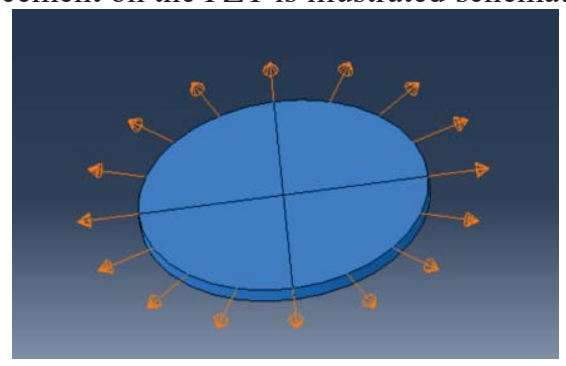

FIGURE 2: Application of the effective radial displacement on the PZT sensor.

Following this process, the signals obtained numerically using the different modelling approaches can be compared with the experimental measurements.

The transient problem of wave propagation requires the use of time integration algorithms. Here time marching is performed explicitly which is only conditionally stable. Crucial for the stability and the accuracy of the solution is the temporal and special discretization of the problem. The time step must satisfy the Courant-Friedrich-Levy (27):

$$
\delta t \leq \frac{l_{\min }}{C_{g}}
$$

where, $\delta t$ is the time-step size, $l_{\min }$ is the smallest element dimension and $C_{g}$ is the group velocity. The element size can be determined through mesh convergence studies. In (28), a minimum of ten nodes per wavelength (NPW) is recommended. In (14), however, larger values are suggested to capture accurately higher wave modes. In this study our focus is mainly the time of arrival of the first wave packets in the frequency range $50-500 \mathrm{kHz}$ that is typical to SHM applications (29). In all subsequent numerical investigations, the element size is set as such that it leads to a minimum of ten NPW while the time step satisfies Eq. 4.

\section{COMPARISON OF THE DIFFERENT MODELS.}

\section{Aluminium Panel}

First the results from the Aluminium plate are presented. This comparison, although simple and well-studied, offers the opportunity to validate the numerical models and evaluate their convergence using an isotropic material. Guided waves are excited at PZT location 1 with central frequency $f_{c}=300 \mathrm{kHz}$ and are recorded at locations 2, 3 and 4 (see Fig. 1). The group velocity of the wave is estimated by computing the time of arrival (TOA) of the signal using its envelope, as illustrated in Fig. 3. The envelope is computed as (30):

$$
S_{\text {env }}=|R-i \mathcal{H}(R)|,
$$

where, $R$ is the signal received and $\mathcal{H}(\cdot)$ is the Hilbert transform. Since the distance between the actuator PZT and the receiver PZT is known, the group velocity can be computed. Using the above process, the estimated group velocity is $C_{g}=5233 \mathrm{~m} / \mathrm{s}$ and the wavelength is $\lambda=0.0175 \mathrm{~m}$. 


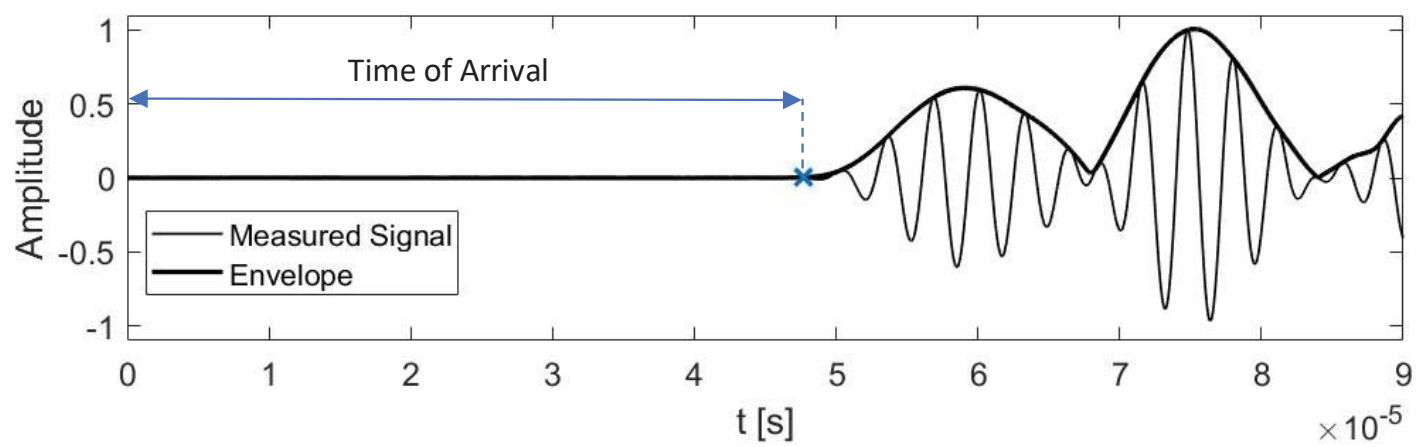

FIGURE 3: Definition of TOA using the signal envelope.

The wave propagation is then simulated numerically in Abaqus. The element size is set to $l_{e}=0.5 \mathrm{~mm}$ which corresponds to $35 \mathrm{NPW}$ and the time step size is set to $\Delta T=6 \cdot 10^{-8} \mathrm{~s}$ that satisfies Eq. 4 . The simulation is repeated for each element type considered. The guided wave propagation for each element type is illustrated schematically in Fig. 4 at different time instants. Differences between the conventional shell with the continuum shell and 3D solid can be attributed to the different representation of the panel boundaries, as also noted in (14). As expected for an isotropic material, the propagation speed is equal along all material directions and the guided waves propagate in a circular pattern.
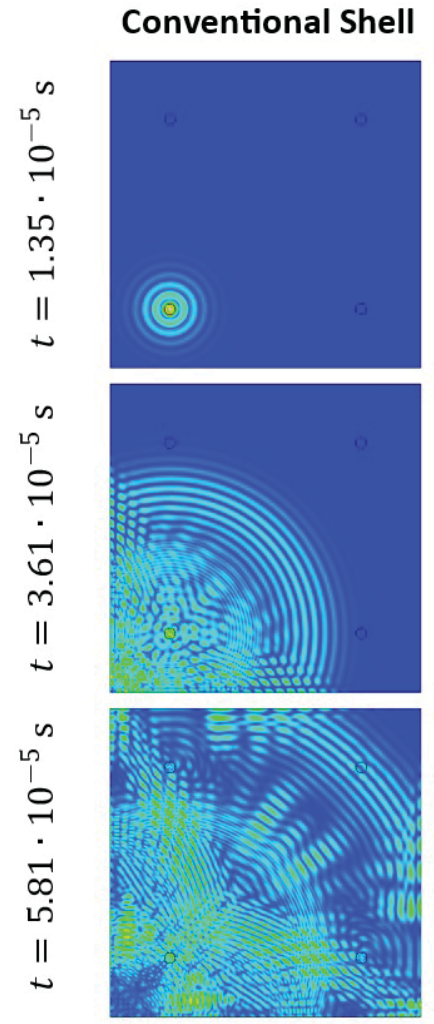

Continuum Shell
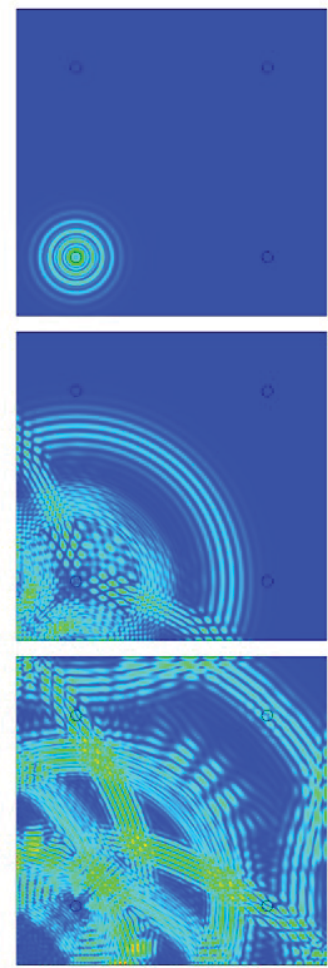

3D Solid
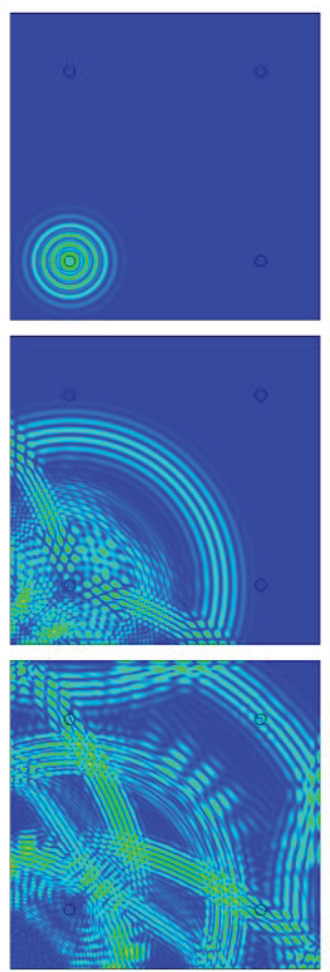

FIGURE 4: Guided wave propagation using the three different element types at time instants $t=1.35 \cdot 10^{-5} s, t=3.61 \cdot 10^{-5} s$ and $t=5.81 \cdot 10^{-5} \mathrm{~S}$.

The signals measured at the sensor locations are compared with the experimental signals in Fig. 5. Due to the material symmetry, the signals recorded at PZT 2 and 4 are the same. In all three cases, the numerical predictions are able to approximate the arrival of the first wave packet with adequate accuracy. It is noted that attenuation phenomena have not been considered in this study. Additionally, the adhesive layer between the PZT wafer and the panel have been modelled as a rigid link. These effects can influence the amplitude of the received waves $(2,31)$. Thus, the amplitudes of the signals reported in Fig. 5 have been normalized for the purposes of the comparison. 

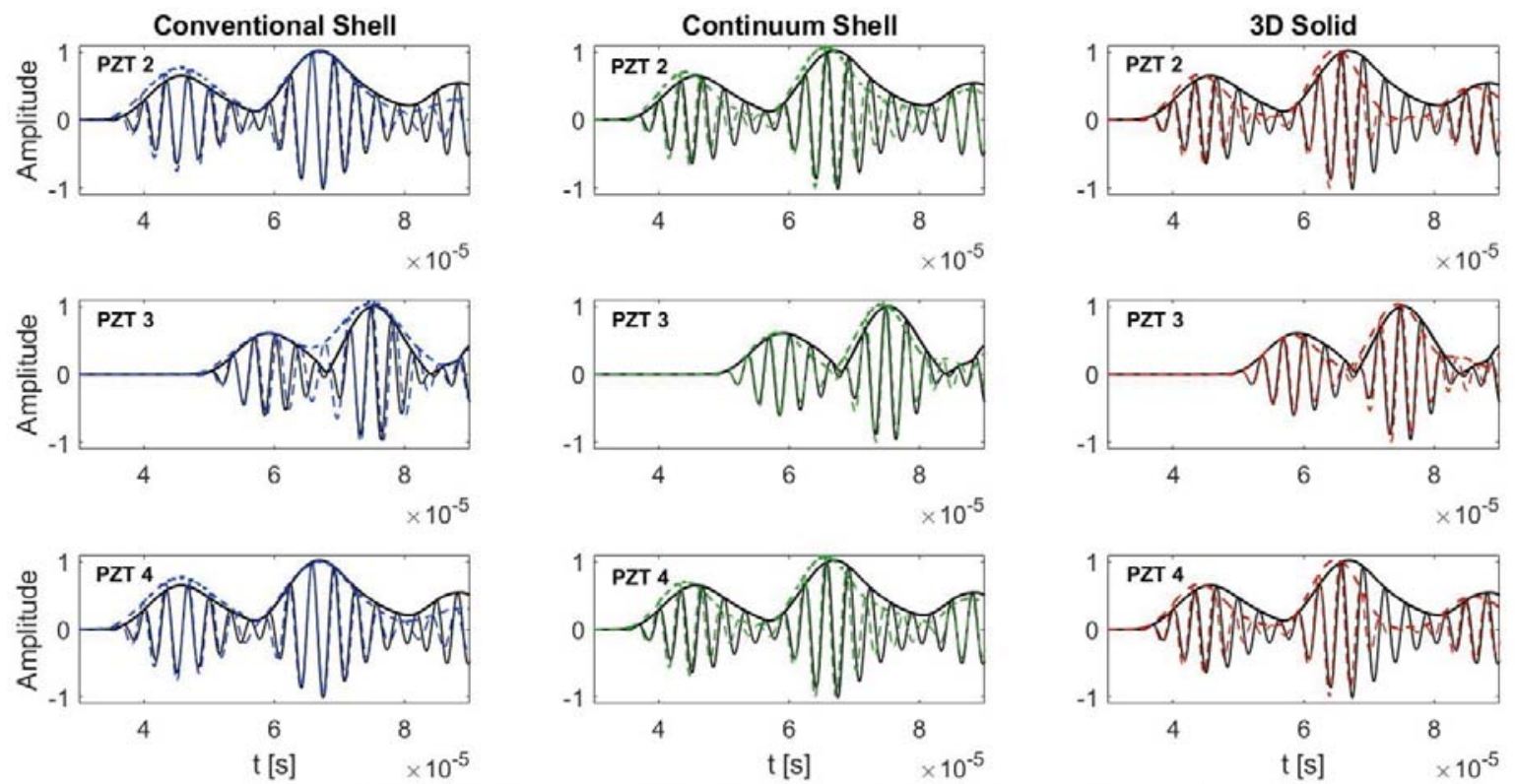

Experimental -- Conventional Shell $-\cdots$ Continuum Shell $---\cdot 3 \mathrm{D}$ Solid

FIGURE 5: Comparison of the received signals at each PZT location with the experimental measurements.

The estimated TOA for each element type is reported in Table 3 along with the resulting group velocity. All three element types are able to make group velocity estimations with small relative error compared to the experimental measurements.

TABLE 3: Comparison of the relative error for the estimation of the group velocity for each element type.

\begin{tabular}{cccc}
\hline & TOA $[\mathrm{s}]$ & $\mathbf{C}_{\mathrm{g}}[\mathrm{m} / \mathrm{s}]$ & Error \% \\
\hline Experimental & $4.7710^{-5}$ & 5247.7 & - \\
Conventional Shell & $4.6810^{-5}$ & 5341.7 & 1.791 \\
Continuum Shell & $4.7410^{-5}$ & 5270.9 & 0.442 \\
3D solid & $4.7810^{-5}$ & 5242.20 & 0.105 \\
\hline
\end{tabular}

\section{Composite Panel}

In this section the results of the composite panel (P2) are presented and discussed. Guided wave propagation in layered composites is complex due to the anisotropic behavior and the different stacking direction of each layer. In special cases, the composite can exhibit behavior similar to that of an isotropic material however, in the general case, the behavior will be anisotropic and the group and phase velocity of each wave mode will depend on the propagation direction (8).

Similar to the isotropic case, our focus is the arrival of the first symmetric $\left(S_{0}\right)$ and antisymmetric $\left(A_{0}\right)$ wave modes. Specifically, an excitation signal with $f_{c}=250 \mathrm{kHz}$ is used for the $S_{0}$ mode while an excitation signal with $f_{c}=50 \mathrm{kHz}$ is used for the $A_{0}$ mode. To ensure adequate NPW for each of the above frequencies, the elements size used is $l_{e}=1 \mathrm{~mm}$ for $f_{c}=50 \mathrm{kHz}$ and $l_{e}=0.5 \mathrm{~mm}$ for $f_{c}=250 \mathrm{kHz}$. Furthermore, when the continuum shell and the solid elements are used, one element for each layer of the composite is used for the through thickness discretization. 

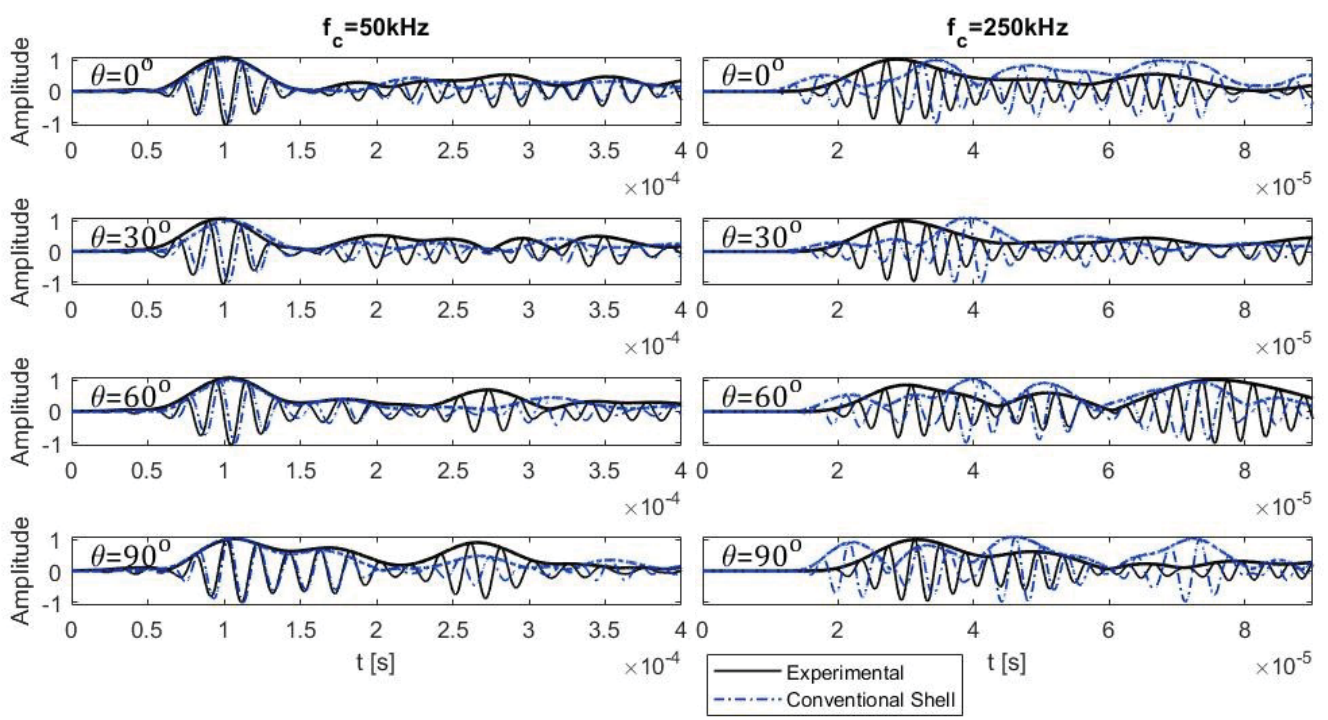

FIGURE 6: Signals recorded at different propagation directions when conventional shell elements are used.
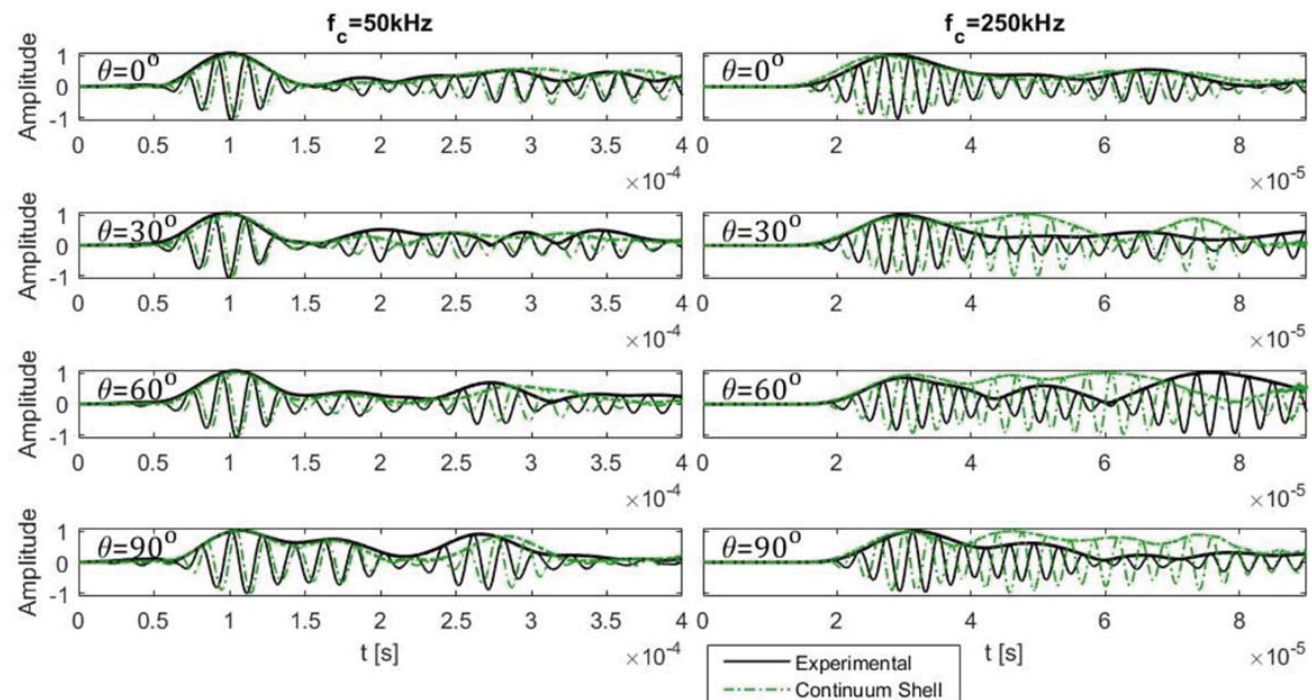

FIGURE 7: Signals recorded at different propagation directions when continuum shell elements are used.

Due to the anisotropic behavior of the material, different propagation directions are used. In all analyses, PZT 1 is the actuator and signals are recorded at locations $2-13$ (see Fig. 1 (B) for the numbering sequence). The signals recorded for propagation directions $\theta=0^{\circ}, \theta=30^{\circ}, \theta=60^{\circ}$ and $\theta=90^{\circ}$ are reported in Fig. 6,7 and 8 , for the conventional shell, continuum shell and $3 \mathrm{D}$ solid elements, respectively. All element types are able to capture accurately the $A_{0}$ wave mode at $f_{c}=50 \mathrm{kHz}$. Discrepancies between the experimental and the numerical signals can be attributed to the boundary conditions of the panel and reflections caused due to the interaction of the propagating wave with the PZTs. The agreement between the experimental and the numerical results is present along all propagation directions.

Differences between the experimental and the numerical signals can be observed when the excitation frequency is increased to $f_{c}=250 \mathrm{kHz}$. In this case, the $S_{0}$ wave mode is dominant and when conventional shell elements are used, the numerical signal arrives earlier than the experimental. When continuum or solid elements are used, the arrival time and the waveform of the first wave packet is better corelated between the experimental and the numerical predictions. Still however, there is a phase difference between the signals. 

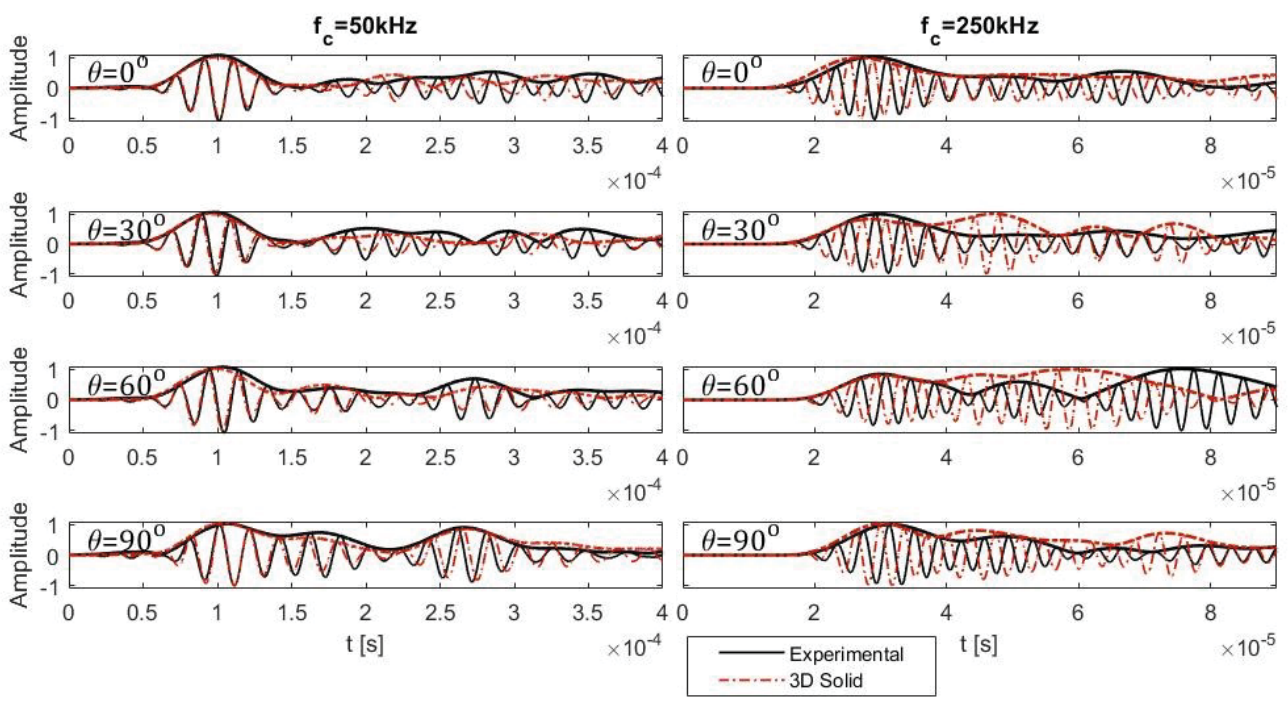

FIGURE 8: Signals recorded at different propagation directions when 3D solid elements are used.

\section{Group Velocity S0@250kHz [m/s]}

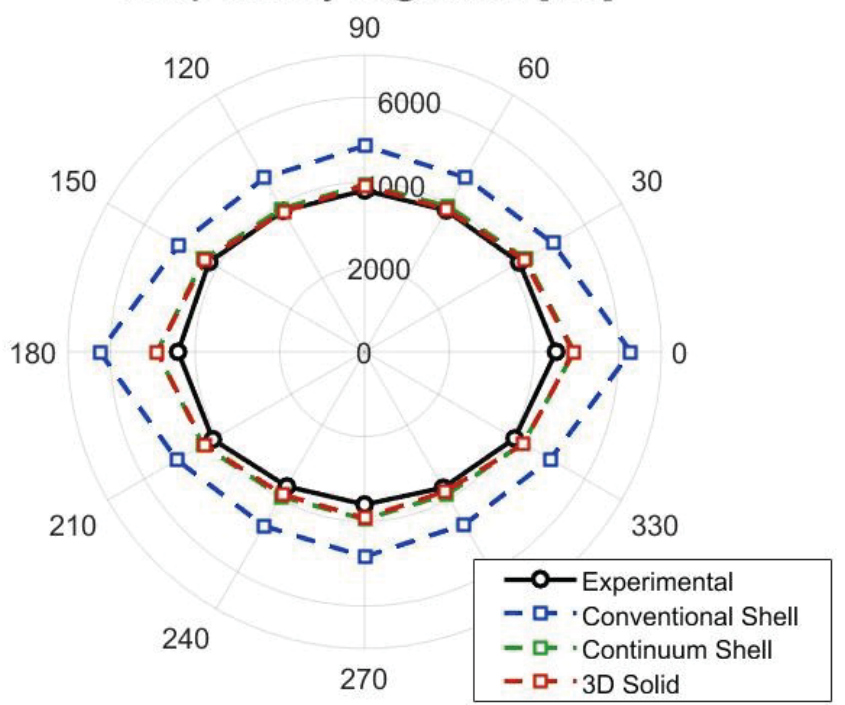

FIGURE 9: Polar plot of the $S_{0}$ group velocity at excitation frequency $f_{c}=250 \mathrm{kHz}$.

The mismatch between the arrival time from the numerical and the experimental predictions is better illustrated by computing the group velocity along each propagation path. The resulting polar plot is reported in Fig. 9. It is apparent that the conventional shell elements overestimate the group velocity of the $S_{0}$ mode. Still, the directional dependence of the wave propagation speed due to the anisotropic behavior of the composite is captured by all element types. The continuum shell and 3D solid elements on the other hand lead to group velocity estimates that are close to the experimental measurements.

The continuum shells and 3D solid elements discretize each layer of the composite explicitly while conventional shell elements use a sectional representation. At higher frequencies and shorter wavelengths, the explicit description of each layer could explain why continuum shells and 3D solid elements remain accurate. Furthermore, the transverse shear assumptions of the conventional shells might underestimate the stress state in the layered composite. In the case of the antisymmetric $A_{0}$ wave mode at lower frequency, the conventional shell elements exhibit comparable accuracy compared to the other two element types. 


\section{CONCLUSIONS AND RECOMMENDATIONS FOR FUTURE WORK}

In the present work guided wave propagation is simulated numerically using conventional shell, continuum shell and 3D solid elements in the commercial finite element software Abaqus. All three element types were able to accurately capture lamb wave propagation for an aluminum panel with excitation frequency $f_{c}=300 \mathrm{kHz}$. The group velocity predictions are in close agreement with the experimental observations. Such estimations can be used for the development, optimization and assessment of fault detection algorithms based on the time of arrival of lamb wave signals (14).

In the case of the layered composite panel, all element types where able to capture the arrival of the antisymmetric $A_{0}$ wave mode for $f_{c}=50 \mathrm{kHz}$ along different propagation directions. The accuracy of the conventional shell elements however deteriorated when the excitation frequency was increased to $f_{c}=250 \mathrm{kHz}$, where the symmetric $S_{0}$ mode is dominant. The results indicate that the conventional shells overestimate the group velocity of the disturbance along all propagation paths. The continuum shell 3D solid elements however remain accurate and lead to group velocity estimates that are closely correlated to the experimental observations.

It must be noted however that the continuum shell and 3D solid elements discretize the thickness of the composite using one element per layer. The conventional shell elements on the other hand, use only a single layer of elements at the reference surface (midplane of the panel). This difference leads to a steep increase of the computational effort required to perform the simulation as the resulting number of equations increases as the number of layers in the composite increases.

The result emphasize that careful selection of the appropriate element type is required. Further studies could investigate the identification of appropriate correction procedures that could enhance the performance of conventional shell elements and broaden their spectrum of applicability. Use of higher order plate theories can circumvent the use of stress correction factors at the cost of additional computational effort (23). Such elements however are not readily available in many commercial software and require the use of user subroutines. A different approach could be the development of compensation procedures for the numerical predictions based on experimental observations.

\section{ACKNOWLEDGEMENTS}

The research leading to these results has gratefully received funding from the European JTICleanSky2 program under the Grant Agreement n॰314768 (SHERLOC).

\section{REFERENCES}

1. Moriot J, Quaegebeur N, Le Duff A, Masson P. A model-based approach for statistical assessment of detection and localization performance of guided wave-based imaging techniques. Struct Health Monit. 2018;17(6):1460-72.

2. Giurgiutiu V. Structural health monitoring: with piezoelectric wafer active sensors. Elsevier; 2007.

3. O’Connor P, Kleyner A. Practical reliability engineering. John Wiley \& Sons; 2012.

4. Michaels JE. Detection, localization and characterization of damage in plates with an in situ array of spatially distributed ultrasonic sensors. Smart Mater Struct. 2008;17(3):035035.

5. Zhao X, Gao H, Zhang G, Ayhan B, Yan F, Kwan C, et al. Active health monitoring of an aircraft wing with embedded piezoelectric sensor/actuator network: I. Defect detection, localization and growth monitoring. Smart Mater Struct. 2007;16(4):1208.

6. Mallardo V, Aliabadi M, Khodaei ZS. Optimal sensor positioning for impact localization in smart composite panels. J Intell Mater Syst Struct. 2013;24(5):559-73.

7. Sharif-Khodaei Z, Ghajari M, Aliabadi M. Determination of impact location on composite stiffened panels. Smart Mater Struct. 2012;21(10):105026.

8. Rose JL. Ultrasonic waves in solid media. 2000;

9. Strikwerda JC. Finite difference schemes and partial differential equations. SIAM; 2004.

10. Cho Y, Rose JL. A boundary element solution for a mode conversion study on the edge reflection of Lamb waves. J Acoust Soc Am. 1996;99(4):2097-109.

11. Li J, Khodaei ZS, Aliabadi MH. Boundary element modelling of ultrasonic Lamb waves for structural health monitoring. Smart Mater Struct. 2020; 
12. Kudela P, Żak A, Krawczuk M, Ostachowicz W. Modelling of wave propagation in composite plates using the time domain spectral element method. J Sound Vib. 2007;302(4-5):728-45.

13. Ostachowicz W, Kudela P, Krawczuk M, Zak A. Guided waves in structures for SHM: the time-domain spectral element method. John Wiley \& Sons; 2011.

14. Sharif-Khodaei Z, Aliabadi M. Assessment of delay-and-sum algorithms for damage detection in aluminium and composite plates. Smart Mater Struct. 2014;23(7):075007.

15. Su Z, Ye L. Identification of damage using Lamb waves: from fundamentals to applications. Vol. 48. Springer Science \& Business Media; 2009.

16. Paćko P, Bielak T, Spencer A, Staszewski W, Uhl T, Worden K. Lamb wave propagation modelling and simulation using parallel processing architecture and graphical cards. Smart Mater Struct. 2012;21(7):075001.

17. Scalerandi M, Agostini V, Delsanto PP, Van Den Abeele K, Johnson PA. Local interaction simulation approach to modelling nonclassical, nonlinear elastic behavior in solids. J Acoust Soc Am. 2003;113(6):304959.

18. Fellinger P, Marklein R, Langenberg KJ, Klaholz S. Numerical modeling of elastic wave propagation and scattering with EFIT—elastodynamic finite integration technique. Wave Motion. 1995;21(1):47-66.

19. Reddy PS, Kesavan R, Ramnath BV. Investigation of mechanical properties of aluminium 6061-silicon carbide, boron carbide metal matrix composite. Silicon. 2018;10(2):495-502.

20. Oñate E. Structural analysis with the finite element method. Linear statics: volume 2: beams, plates and shells. Springer Science \& Business Media; 2013.

21. Reddy JN. Mechanics of laminated composite plates and shells: theory and analysis. CRC press; 2003.

22. Mindlin R. Influence of rotatory inertia and shear on flexural motions of isotropic, elastic plates. J Appl Mech. 1951;18:31-8.

23. Reddy JN. A simple higher-order theory for laminated composite plates. 1984;

24. Abaqus Analysis User's Guide, Dassault Systèmes Simulia Corp. 2016.

25. Samaratunga D, Jha R. Lamb wave propagation simulation in smart composite structures. In 2012. p. 1-11.

26. Yang C, Ye L, Su Z, Bannister M. Some aspects of numerical simulation for Lamb wave propagation in composite laminates. Compos Struct. 2006;75(1-4):267-75.

27. Duczek S, Joulaian M, Düster A, Gabbert U. Numerical analysis of Lamb waves using the finite and spectral cell methods. Int J Numer Methods Eng. 2014;99(1):26-53.

28. Alleyne D, Cawley P. A two-dimensional Fourier transform method for the measurement of propagating multimode signals. J Acoust Soc Am. 1991;89(3):1159-68.

29. Aliabadi MF, Khodaei ZS. Structural health monitoring for advanced composite structures. Vol. 8. World Scientific; 2017.

30. Salmanpour M, Sharif Khodaei Z, Aliabadi M. Guided wave temperature correction methods in structural health monitoring. J Intell Mater Syst Struct. 2017;28(5):604-18.

31. Gresil M, Giurgiutiu V. Prediction of attenuated guided waves propagation in carbon fiber composites using Rayleigh damping model. J Intell Mater Syst Struct. 2015;26(16):2151-69. 